\title{
THE CLINICAL VALUE OF THE KOLMER COMPLEMENT-FIXATION TEST FOR SYPHILIS *
}

CHARLES H. DET. SHIVERS, M.D.

Assistant Professor of Syphilology, Graduate School of Medicine, University of Pennsylvania; Chief of the U. S. Public Health Service for the Treatment of Genito-Urinary Diseases

ATLANTIC CITY, N. J.

The complement-fixation reaction has proved of great value in the diagnosis of syphilis and as a guide to specific therapy. Unfortunately, the original method of Wassermann and his co-workers lacks sufficient sensitiveness; and, as a result of attempts to improve the technic, a large number of modifications have come into use. These different methods vary in sensitiveness and practical specificity; for that reason portions of the blood of the same person examined in different laboratories may yield varying results, lead to considerable confusion, and result in undetermining the fundamental and high values of the complement-fixation test in syphilis.

For these and additional reasons a demand for standardization of the Wassermann reaction has arisen, and a good start in this direction has been made by the exhaustive investigations of Kolmer and his colleagues. ${ }^{1}$ These studies have resulted in the elaboration of a new antigen $^{2}$ and a new technic ${ }^{3}$ which Kolmer believes may fulfil the aims of increased sensitiveness and practical specificity, as well as yield a true quantitative reaction of greater value as a serologic guide to treatment than afforded by present methods. The new method has been employed by Kolmer in the Polyclinic Laboratories of the Graduate School of Medicine of the University of Pennsylvania for the last two years, and recently Schamberg and Klauder ${ }^{4}$ have published the results observed by them in about 2,000 tests. These authors found the new Kolmer method distinctly more sensitive than the older method and highly specific, inasmuch as false positive reactions in nonsyphilitic subjects were not observed. The new method was found to harmonize better with the clinical findings, and its quantitative character

* Read before the Philadelphia Urological Society, April 24, 1922.

1. Series of thirty-two papers being published in the Am. J. Syphilis, beginning 3:1, 1919.

2. Am. J. Syphilis 6:74, 1922.

3. Am. J. Syphilis 6:82, 1922.

4. Medical Clinics of North America, Philadelphia, W. B. Saunders Company, 1921 , p. 667. 
permitted a better gage of the effects of antisyphilitic treatment than yielded by older methods.

\section{AUTHOR'S INVESTIGATIONS}

I have had 320 tests made by Dr. Kolmer with the serums of private patients whom he was able to study clinically with particular care. Each serum was examined by the new method and likewise by Dr. Kolmer's older three antigen method. All serums yielding a positive reaction with the new method either had a previous history of syphilitic infection or presented clinical evidences of the disease. In no instance were falsely positive reactions observed.

The new test was frequently positive early in the primary stages when the old method reacted negatively even with cholesterolized antigens. The new test likewise was the first to detect a relapsing case and the last to give a negative reaction during treatment.

\section{REPORT OF CASES}

CASE 1.-H. L., a man, aged 51, had had a primary lesion twenty years ago. He was under treatment for six months at that time with mercury and iodids. Two years ago a Wassermann test was made and found to be four plus. Following this examination he received four injections of arsphenamin, $0.6 \mathrm{gm}$. each. At present there is no clinical evidence of syphilis. The Kolmer method gave a moderately positive reaction, while the old method was negative. This illustrates the value of the new test in detecting reagin ${ }^{8}$ in the blood of a patient with latent asymptomatic syphilis.

CASE 2.-F. C., a man, aged 22 , gave no history of a primary infection. He had had one attack of gonorrhea with many recurrences. Following his first Neisserian infection, he noticed falling of the hair. A Wassermann test was made at that time and found to be four plus. He was then given twenty-four injections of neo-arsphenamin. At present there are no symptoms except the loss of hair. Examination was negative. The Wassermann reaction made on Aug. 30, 1920, was weakly positive with the Kolmer method, and negative with the old method. After ten injections consisting of $0.6 \mathrm{gm}$. each of arsphenamin, at weekly intervals, combined with inunctions of mercury, 1 dram $(3.9 \mathrm{gm}$.) four times a week, the Wassermann test was again made and showed negative in both tests. Without further treatment a third Wassermann test was made on Feb. 25, 1921, which was weakly positive with the new method and negative with the old. The Kolmer method, as illustrated in this case, is the last to show a negative reaction while the patient is under treatment, and the first to detect a relapsing positive.

CASE 3.-A. J., a man, aged 52, gave a history of a primary lesion thirty-two years ago. Secondary symptoms followed. No treatment was received by the patient at this time. The only symptoms complained of when the patient reported were severe frontal and occipital headaches. This man had been treated previously for sinus infection. Examination showed the pupils to be slightly irregular, with a sluggish reaction to light. The Kolmer method

5. Term designated by Neisser for the reacting substance in the blood serum. 
showed a moderately positive reaction; the old test was weakly positive. This patient was entirely relieved of his head symptoms after a few injections of neo-arsphenamin. In this case the test gave one more assurance of the correctness of the clinical diagnosis. A spinal Wassermann test was not made.

CASE 4.-M. S., a woman, aged 45 , gave no history of any infection. The first Wassermann test made by the health department of another city showed a three plus reaction with all three antigens. This patient had received twelve injections before the Kolmer technic was used. The Wassermann test made on Oct. 15, 1920, showed a moderately positive reaction with the Kolmer method and a weakly to moderately positive reaction with the old method. Fifteen injections of neo-arsphenamin were then given at irregular intervals, and the Wassermann test on Nov. 3, 1921, was weakly positive with the Kolmer method and negative with the old. This persistent positive reaction with the new test indicates the necessity for more treatment, at the same time showing improvement in the higher dilutions of the patient's serum.

CASE 5.-S. R., a man, aged 23, reported for treatment on Aug. 24, 1919, with a positive previous history of syphilis. The only symptom he complained of was general weakness: The physical examination was negative. The Wassermann test made at this time showed a four plus reaction with all three antigens with the old test. Twenty-four injections of arsphenamin, $0.6 \mathrm{gm}$. each, were given, together with mercury by inunctions and potassium iodid. The Wassermann test made on Nov. 9, 1920, showed a moderately positive reaction with Kolmer's new method, and a negative reaction with the old. Several more injections of arsphenamin were then given; the blood examination following these treatments was negative with both tests. This case also showed the slowness of the new method to become negative while the patient is under treatment.

CASE 6.-S. M., a man, aged 28, had a primary lesion in June, 1917, or three years before he first reported to me for treatment. He had received six injections of arsphenamin at that time; there was no history of secondary symptoms. One year ago he noticed that the vision was blurred in his left eye. The oculist reported retinal hemorrhages in the macular region. At this time he received twelve injections of neo-arsphenamin at weekly intervals. Spinal fluid and blood Wassermann tests on Nov. 30, 1920, showed a weakly positive reaction with the new method and a negative reaction with the old. In addition, in the spinal fluid examination the colloidal gold test showed the syphilitic curve; the cell count was normal. This is a case of neurosyphilis of the vascular type; in which immediate and intensive treatment was essential; yet the blood and spinal fluid reactions were negative with the old method.

CASE 7.-W. H. Mc., a man, aged 20, denied all venereal infections. He consulted a physician on account of enlargement of the inguinal glands. Examination disclosed a poorly nourished man with general glandular enlargement. No other signs of the disease were present. Blood was taken for a Wassermann test on April 4, 1921. The Kolmer method showed a weakly positive reaction, the old method was negative. It was later discovered that the father of this boy contracted syphilis two years before the patient was born, and that he had received insufficient treatment. This is a case of congenital syphilis in which the old method was negative with all three antigens, and in which the clinical findings were not sufficient to make a diagnosis.

CASE 8.-F. H., a man, aged 28 , had a primary lesion of two weeks' duration; the dark field was positive. The blood for a Wassermann test was taken on the fifteenth day, and the result obtained is very interesting. The Kolmer 
method showed a strongly positive reaction, while the old method was weakly positive, giving evidence of the earlier positives with the new method. After giving seventeen injections of neo-arsphenamin, $0.9 \mathrm{gm}$. each, with a course of mercury, the Wassermann test was again made and showed a weakly positive reaction with the Kolmer method, and a negative reaction with the old method. The new test later became negative after the patient had received more treatments.

CASE 9.-J. S., a man, aged 28, had a primary lesion during the early part of September, 1921. Diagnosis was made by dark-field examination. Seventeen injections of neo-arsphenamin, $0.9 \mathrm{gm}$. each, were given at stated intervals. Treatment was instituted about two weeks after the appearance of the sore. The Wassermann test made by the new method was strongly positive and by the old method moderately positive. Mercury was given by inunctions together with potassium iodid. The blood Wassermann test was made again on Dec. 6, 1921, and was negative with both tests. $U_{p}$ to this time twelve of the seventeen injections had been given. On Jan. 17, 1922, the patient, while walking along the street, had a convulsion, followed by a period of unconsciousness lasting several hours. Another attack similar to the first occurred on Feb. 4, 1922. The blood continued negative with both tests. On Feb. 10, 1922, spinal fluid was withdrawn for an examination. The fluid came out with considerable pressure but was clear. The report showed a cell count of 40 per cubic millimeter. The colloidal gold reaction was negative. The Kolmer method showed a one plus, or weakly positive reaction, while the old test was negative. Treatment was again started with weekly injections of neo-arsphenamin, $0.9 \mathrm{gm}$. each, followed every other week by spinal drainage. The patient has shown wonderful improvement as to nervousness, mentality and strength. This case is of special interest because it shows that Dr. Kolmer's new method is even more sensitive than the colloidal gold reaction in a case of clinical neurosyphilis of the meningeal type.

The accompanying table briefly summarizes cases from this series in which the two methods yielded different reactions; in every instance the results of the new tests have proved correct and consistently indicative of superior sensitiveness and specificity.

\section{SUMMARY}

From the standpoint of the practitioner, the complement-fixation test for syphilis should be as sensitive as is consistent with specificity. There can be no difference of opinion in regard to the absolute necessity for practical specificity, that is, extreme sensitiveness is undesirable if it means the possibility of securing falsely positive reactions. This point has been kept clearly in mind by Dr. Kolmer, and his efforts to improve the sensitiveness of the reaction without at the same time running the risk of falsely positive reactions has evidently been realized in my experience. This is due in large part to the remarkable antigen being employed which in a dose of 10 units is forty or more times less than the anticomplementary unit.

In my experience this increased sensitiveness of the complementfixation reaction for syphilis is highly desirable for the early diagnosis 
The Relative Efficiency of the Kolmer Complement-Fixation TEST FOR SYPHILIS

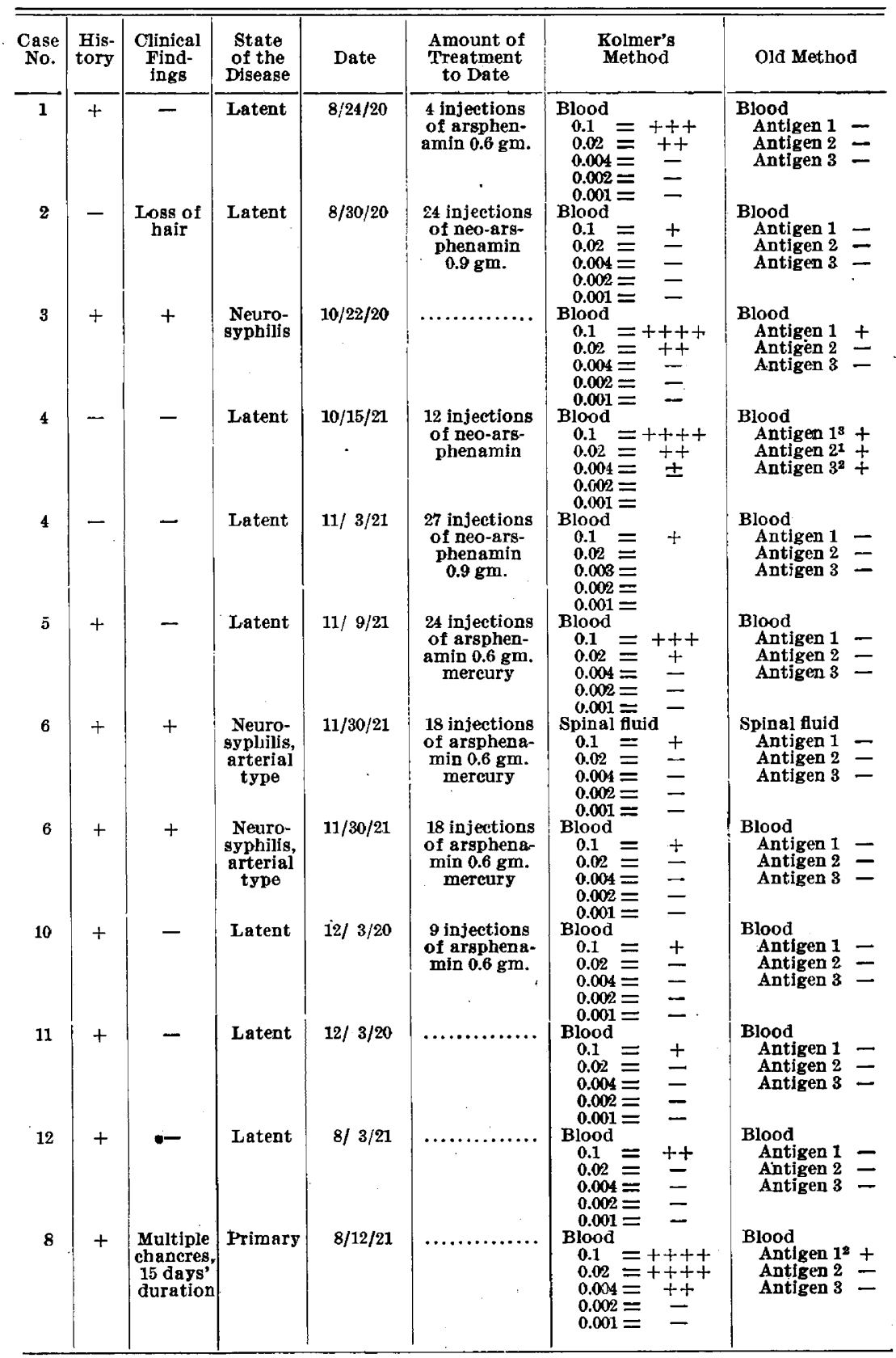


The Relative Efficiency of the Kolmer Complement-Fixation

TEST For SyphILIS-(Continued)

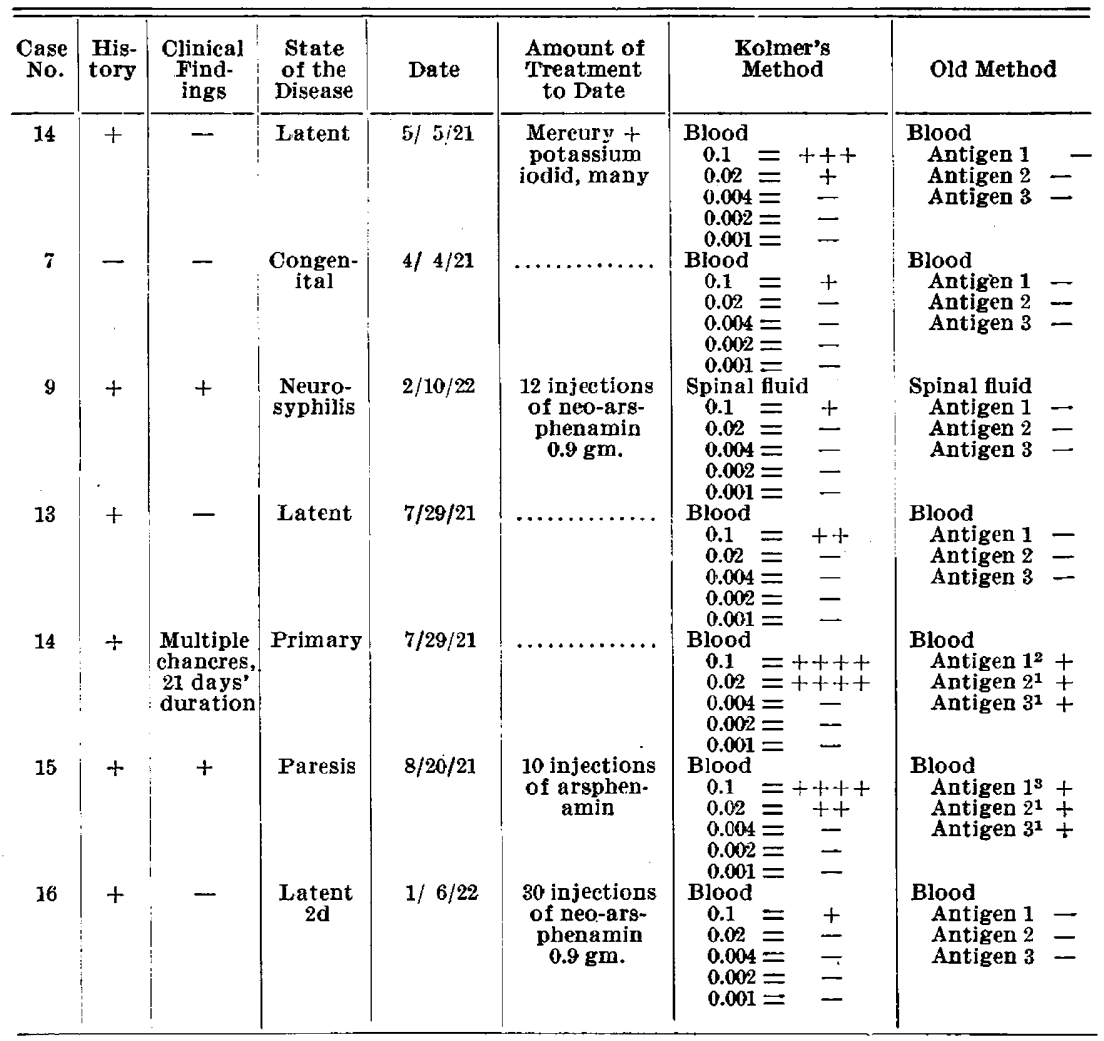

of syphilis, and more especially for diagnosis in the latent and tertiary stages and in congenital syphilis. Furthermore, this sensitive method requires more thorough treatment for the eradication of positive reactions, and I regard this as an important point in view of the tendency to undertreat this disease. Negative reactions with the new test have been secured by treatment, but considerably more treatment is required for this result by the new method than by older methods.

In my opinion the complement-fixation reaction in syphilis is not too delicate, but not delicate enough; as pointed out by Kolmer, ${ }^{6}$ the reaction may yield falsely negative reactions in cases of syphilis in which the spirochetes are present in such few numbers or in such a condition of latency or quiescence that sufficient amounts of "reagin" are not being produced in the blood and spinal fluid to yield positive reactions. For this reason the complement-fixation reaction for syphilis

6. Kolmer, John A.: Standardization of the Wassermann Reaction, J. A. M. A. 77:776 (Sept. 3) 1921. 
is still apt to err on the negative side and is worthy of further efforts toward rendering the test even more sensitive, providing, always that it is safely kept within the bounds of practical specificity.

The quantitative feature of the new test secured by testing each serum in varying amounts had likewise proved useful in my experience. Of course the question of whether a serum or spinal fluid does or does not yield a positive reaction is of primary importance, and treatment aims to render the reaction negative. But during the course of treatment an accurate gage of the progress of reduction of "reagin" in the blood is of value and offers distinct encouragement to both physician and patient.

The new method has likewise proved of superior sensitiveness and reliability for the examination of spinal fluids, and in a number of cases it has yielded true positive reactions with fluids from cases of early neurosyphilis when the older method has yielded negative reactions.

\section{CONCLUSIONS}

1. The writer has employed the Kolmer complement-fixation test for syphilis in 320 cases-some with a definite history of specific infection and presenting clinical evidences of syphilis, others with negative histories and presenting no signs or symptoms of syphilis.

2. The new method has proved a very sensitive and reliable test, much more so than the old method, being frequently positive while the iatter is negative.

3. The new method has yielded earlier positive reactions in primary syphilis than other methods, and is much slower in becoming negative when the patient is under treatment than the old method.

4. A negative reaction with the Kolmer method is of more value when giving a prognosis.

5. The new Kolmer method has not yielded falsely positive reactions in nonsyphilitic persons.

6. The method has been proved more valuable in aiding a diagnosis of congenital syphilis in obscure cases than the old method.

7. The new method marks a distinct and valuable advance in the serum diagnosis of syphilis, being much more sensitive and at the same time yielding no falsely positive reactions. 